\title{
Inhibition of Monocarboxylate Transporter 2 in the Retrotrapezoid Nucleus in Rats: A Test of the Astrocyte- Neuron Lactate-Shuttle Hypothesis
}

\author{
Joseph S. Erlichman, ${ }^{1}$ Amy Hewitt, ${ }^{1}$ Tracey L. Damon, ${ }^{1}$ Michael Hart, ${ }^{1}$ Jennifer Kurascz, ${ }^{1}$ Aihua Li, ${ }^{2}$ and James C. Leiter ${ }^{2}$ \\ ${ }^{1}$ Department of Biology, St. Lawrence University, Canton, New York 13617-1475, and ²Department of Physiology, Dartmouth Medical School, Lebanon, \\ New Hampshire 03756
}

\begin{abstract}
The astrocyte-neuronal lactate-shuttle hypothesis posits that lactate released from astrocytes into the extracellular space is metabolized by neurons. The lactate released should alter extracellular $\mathrm{pH}(\mathrm{pHe})$, and changes in $\mathrm{pH}$ in central chemosensory regions of the brainstem stimulate ventilation. Therefore, we assessed the impact of disrupting the lactate shuttle by administering $100 \mu \mathrm{m} \alpha$-cyano-4-hydroxycinnamate (4-CIN), a dose that blocks the neuronal monocarboxylate transporter (MCT) 2 but not the astrocytic MCTs (MCT1 and MCT4). Administration of 4-CIN focally in the retrotrapezoid nucleus (RTN), a medullary central chemosensory nucleus, increased ventilation and decreased $\mathrm{pHe}$ in intact animals. In medullary brain slices, 4-CIN reduced astrocytic intracellular $\mathrm{pH}$ (pHi) slightly but alkalinized neuronal pHi. Nonetheless, pHi fell significantly in both cell types when they were treated with exogenous lactate, although $100 \mu \mathrm{m} 4$-CIN significantly reduced the magnitude of the acidosis in neurons but not astrocytes. Finally, 4-CIN treatment increased the uptake of a fluorescent 2-deoxy-D-glucose analog in neurons but did not alter the uptake rate of this 2-deoxy-D-glucose analog in astrocytes. These data confirm the existence of an astrocyte to neuron lactate shuttle in intact animals in the RTN, and lactate derived from astrocytes forms part of the central chemosensory stimulus for ventilation in this nucleus. When the lactate shuttle was disrupted by treatment with 4-CIN, neurons increased the uptake of glucose. Therefore, neurons seem to metabolize a combination of glucose and lactate (and other substances such as pyruvate) depending, in part, on the availability of each of these particular substrates.
\end{abstract}

Key words: astrocytes; neurons; lactate shuttle; metabolism; ventilation; $\mathrm{pH}$

\section{Introduction}

Magistretti and Pellerin (1999) proposed that an astrocyticneuronal lactate shuttle exists in which glutamate, released from neurons during synaptic activity, is taken up by astrocytes in a sodium-coupled process, and the ensuing sodium-dependent activation of the Na-K-ATPase triggers glucose uptake, which results in the rapid formation and release of lactate from astrocytes via monocarboxylate transporter (MCT) 1 (MCT1) and MCT4. The lactate released by astrocytes into the extracellular space is, according to this hypothesis, transported into neurons by MCT2 and used to fuel activity-dependent energy demands. A family of MCTs transports lactate, pyruvate, and ketone bodies across biological membranes (Halestrap and Price, 1999), and MCT1, MCT2, and MCT4 have been identified in the CNS. MCT1 and MCT4 are expressed in astrocytes, and MCT2 is expressed in neurons (Bröer et al., 1997; Gerhart et al., 1997; Pierre et al., 2002; Debernardi et al., 2003). Because MCT2 has a lower $K_{\mathrm{m}}$ for lactate $(\sim 0.7 \mathrm{~mm})$ than the $K_{\mathrm{m}}$ for either MCT1 or MCT4 $(\sim 5-7$ and

\footnotetext{
Received Dec. 8, 2007; revised Feb. 14, 2008; accepted March 18, 2008.

This work was supported by National Institutes of Health Grant HL-71001 and National Science Foundation Grant IOB-0517698.

Correspondence should be addressed to James C. Leiter at the above address. E-mail: james.c.leiter@dartmouth.edu.

D01:10.1523/JNEUROSCI.5430-07.2008

Copyright $\odot 2008$ Society for Neuroscience $\quad$ 0270-6474/08/284888-09\$15.00/0
}

28-35 mM, respectively) (Bröer et al., 1997, 1999; Poole and Halestrap, 1997; Halestrap and Price, 1999; Manning Fox et al., 2000), the cell-specific distribution of these transporters between astrocytes and neurons seems designed to move lactate from astrocytes to neurons.

One consequence of shuttling lactate between astrocytes and neurons is the effect of lactate on extracellular and intracellular $\mathrm{pH}$. The buffering power of the extracellular space is low (Pappenheimer et al., 1965), and changes in either lactate efflux or influx in astrocytes or neurons can result in significant reciprocal fluctuations in $\mathrm{pH}$. Blood and brain tissue $\mathrm{pH}$ are maintained at constant levels by central respiratory chemoreceptors located primarily in the medulla. These chemosensors detect $\mathrm{pH}$ and adjust ventilatory drive to alter blood and tissue levels of $\mathrm{CO}_{2}$ and thereby regulate $\mathrm{pH}$. The retrotrapezoid nucleus (RTN) in the ventral medulla contains chemosensory neurons that provide an excitatory $\mathrm{pH}$-dependent drive to breathing. RTN neurons, many of which are glutamatergic (Mulkey et al., 2004), increase their activity as the $\mathrm{CO}_{2}$ rises and the $\mathrm{pH}$ falls (Ritucci et al., 2005). In the current study, we used the respiratory effects of changes in extracellular $\mathrm{pH}(\mathrm{pHe})$ and intracellular $\mathrm{pH}(\mathrm{pHi})$ to test aspects of the astrocyte-neuronal lactate-shuttle hypothesis (ANLSH). Much of the support for the lactate-shuttle hypothesis comes from cultured cells (Kimmelberg, 2004), and we were therefore interested in testing the lactate shuttle hypothesis in 
vivo or in situ in brain slices. To that end, we first examined the ventilatory effects of focally inhibiting MCT2 in the RTN in vivo, and we measured pHe to determine whether inhibiting MCT2 altered pHe within the RTN. Second, we tested the hypothesis that inhibition of MCT2 and impaired lactate transport would lead to accumulation of lactate in astrocytes and a fall in pHi but prevent uptake of lactate in neurons and alkalinize neuronal pHi. Finally, we tested the hypothesis that inhibition of neuronal lactate uptake would be associated with a reciprocal increase in neuronal glucose uptake.

\section{Materials and Methods}

The St. Lawrence University Animal Review Committee approved this study, and all procedures were performed in accordance with the $\mathrm{Na}$ tional Institutes of Health Guide for the Care and Use of Laboratory Animals.

In vivo studies of MCT inhibition. Adult Sprague Dawley rats of either sex 7-11 weeks of age weighing between 250 and $350 \mathrm{~g}$ were used for this study. Animals were kept on a $12 \mathrm{~h}$ light/dark cycle and provided food and water ad libitum. Each rat was prepared for the study by placing a push-pull cannula (Plastics One, Roanoke, VA) unilaterally in the RTN. To do this, each rat was anesthetized with xylazine $(6 \mathrm{mg} / \mathrm{kg}$, i.p. $)$ followed by ketamine $\cdot \mathrm{HCl}(70 \mathrm{mg} / \mathrm{kg}$, i.m.) and secured in a stereotaxic apparatus (David Kopf Instruments, Tujunga, CA). A midline incision was made, a hole was drilled in the skull $5 \mathrm{~mm}$ caudal to lambda and 2.2 $\mathrm{mm}$ from the midline (left side), and a push-pull guide cannula [inner diameter (I.D.), $0.29 \mathrm{~mm}$; outer diameter (O.D.), $0.56 \mathrm{~mm}$ ] was inserted through the burr hole. Two anchor screws were placed adjacent to the cannula, and the guide cannula and the anchor screws were attached to the skull with cranioplast (Plastics One). The scalp was sutured together up to the border of the guide cannula. A dummy obturator was screwed into the guide cannula to maintain patency of the lumen during the recovery period and between experiments. The obturator extended 0.3 $\mathrm{mm}$ beyond the end of the guide cannula. Approximately 1 week after surgery, ventilatory measurements were made in each animal. In previous work, we found that ventilation was unaffected by the surgery or by perfusion of the RTN with artificial CSF (aCSF) alone.

CSF solutions and solution delivery. aCSF contained the following salts (in mM): $124 \mathrm{NaCl}, 3 \mathrm{KCl}, 2.4 \mathrm{CaCl}_{2}, 1.3 \mathrm{MgSO}_{4}, 1.24 \mathrm{KH}_{2} \mathrm{PO}_{4}, 26$ $\mathrm{NaHCO}_{3}$, and 10 glucose. Before delivery, aCSF was equilibrated with $95 \% \mathrm{O}_{2}$ and $5 \% \mathrm{CO}_{2}$, warmed to $37^{\circ} \mathrm{C}$, and loaded into $3 \mathrm{ml}$ plastic syringes. The $\mathrm{pH}$ of all solutions was 7.48 . Solutions were delivered to the RTN using a push-pull syringe pump (World Precision Instruments, Sarasota, FL). Flow was directed through polyethylene tubing that was connected to an inner delivery cannula (Plastics One) (I.D., $0.1 \mathrm{~mm}$; O.D., $0.2 \mathrm{~mm}$ ). Before perfusate delivery, the line and inner cannula were flushed with aCSF or aCSF plus the drug to be tested, and the solution was perfused throughout each experiment. After the dead space of the inner cannula was filled, the cannula was screwed into the guide cannula, and the return vacuum line was connected. Like the dummy cannula, the inner delivery cannula extended $0.3 \mathrm{~mm}$ beyond the end of the guide cannula. The flow through the cannula was $0.12 \mathrm{ml} / \mathrm{h}$. Push-pull cannulas resemble microdialysis in that the amount of tissue exposed to the agent is restricted, and the concentration of the test agent remains constant at the tissue adjacent to the probe throughout the perfusion period.

Ventilatory responses to carbon dioxide. Body weight and barometric pressure were recorded before each experiment. Each animal was placed in the plethysmograph and allowed to accommodate to the chamber for 30 min. Perfusion of the push-pull cannula began at the start of the 30 min accommodation period in the plethysmograph and was continued throughout each experiment. During the experiment, each animal was exposed serially to increasing levels of humidified $\mathrm{CO}_{2}$ in the plethysmograph beginning with room air and progressing in $2 \%$ increments to a final fractional inspired $\mathrm{CO}_{2}\left(\mathrm{FICO}_{2}\right)$ of $8 \%$. Animals were maintained at each level of $\mathrm{CO}_{2}$ for $\sim 10 \mathrm{~min}$ after the stabilization of $\mathrm{CO}_{2}$ levels within the plethysmograph chamber. The chamber had a volume of $\sim 1 \mathrm{~L}$, and the rate of airflow through the box was $1.25 \mathrm{~L} / \mathrm{min}^{-1}$. Stable $\mathrm{CO}_{2}$ values within the chamber were achieved in $2-3 \mathrm{~min}$. The chamber carbon dioxide concentration (CD3A; Applied Electrochemistry, Naperville, IL) and pressure (PT5; Grass Instruments, Quincy, MA) were measured continuously, and the signals were low-pass filtered at $10 \mathrm{~Hz}$, digitized at 20 $\mathrm{Hz}$, smoothed using a moving-average transformation, and stored on a personal computer (World Precision Instruments; Biopac Systems, Goleta, CA). Both the chamber and rat body temperature were measured at the end of each step change in $\mathrm{CO}_{2}$ concentration. Body temperature was measured using an infrared sensor (C1600; Linear Laboratories, Freemont, CA). For tidal volume calculations, the measured body temperature was corrected to rectal temperature based on previous experiments in which these body temperatures were determined by infrared emissivity and rectal temperature measured simultaneously. The average pressure change of each breath, which reflects the magnitude of the tidal volume, was determined from 100 breaths during the last 2 min of each test period (i.e., room air or $\mathrm{CO}_{2}$ ). The frequency of breathing was measured during the last minute of each period. Tidal volume was calculated using the method described by Pappenheimer (1977).

Inhibition of lactate transport. MCT transport was inhibited using $\alpha$-cyano-4-hydroxy-cinnamate (4-CIN) stock solution dissolved in $0.1 \%$ DMSO and used at a final concentration of 100 or $500 \mu \mathrm{M}$ (SigmaAldrich, St. Louis, MO) dissolved in aCSF. 4-CIN is a competitive, nontransportable inhibitor of MCTs. The $\mathrm{IC}_{50}$ value (the concentration of drug required to reduce lactate transport by one-half) is $425 \mu \mathrm{M}$ for MCT1, $900 \mu \mathrm{M}$ for MCT4, and $24 \mu \mathrm{M}$ for MCT2 (Bröer et al., 1999). A $500 \mu \mathrm{M}$ concentration of 4 -CIN would be expected to inhibit MCT1 and MCT4 transport by no more than $50 \%$, whereas transport by MCT2 would be virtually eliminated ( $500 \mu \mathrm{M}$ is $\sim 21$ times the $\mathrm{IC}_{50}$ ). A $100 \mu \mathrm{M}$ concentration of 4-CIN should inhibit monocarboxylate transport by MCT1 and MCT4 by $<13 \%$ while eliminating monocarboxylate transport by MCT2 $\left(100 \mu \mathrm{M} 4\right.$-CIN is approximately four times the $\mathrm{IC}_{50}$ MCT2). In the in vivo experiments, we focally perfused the RTN unilaterally with normal aCSF ( $\mathrm{pH} 7.48$ ) or aCSF plus $100 \mu \mathrm{M} 4$-CIN to determine whether inhibition of MCT2 increased ventilation at each level of $\mathrm{CO}_{2}$ tested (Hewitt et al., 2004; Parisian et al., 2004). Each perfusate was tested on a separate day, and at least $1 \mathrm{~d}$ separated each perfusate trial. The order in which the perfusates were delivered was randomized within each animal.

In vivo tissue $\mathrm{pH}$ measurements. Tissue $\mathrm{pH}$ measurements were made using a custom-fabricated electrode (Beetrode; WPI) placed in the RTN through a steel guide cannula located just dorsal to the retrotrapezoid nucleus. After measuring $\mathrm{pHe}$ in the control state while the animal breathed room air, MCT function was impaired by injecting 200-300 nl of $100 \mu \mathrm{M} 4$-CIN unilaterally into the RTN through the guide tube (a larger volume of 4-CIN was administered, because we could not continuously perfuse and measure $\mathrm{pHe}$ simultaneously). The $\mathrm{pH}$ electrode was placed in the RTN, and the pHe adjacent to the electrode was recorded in millivolts for $10 \mathrm{~min}$ in intact awake rats. In separate experiments, the $\mathrm{pH}$ electrode was placed into the RTN via the guide cannula, and the $\mathrm{FICO}_{2}$ of the animal was increased to $5 \%$ while the $\mathrm{pHe}$ was measured for $10 \mathrm{~min}$ in intact awake rats. The millivolt readings occasionally drifted for no apparent reason, and measurements in which the control pHe values before and after each treatment differed by $>2 \mathrm{mV}$ ( $0.034 \mathrm{pH}$ units) were excluded. Stable pHe measurements were recorded as millivolts with pencil and paper during each condition and converted to $\mathrm{pH}$ using a calibration factor measured in vitro. The $\mathrm{pH}$ electrodes were calibrated in vitro with standard $\mathrm{pH}$ buffers ( $\mathrm{pH} 4,7$, and 10 ) at $38^{\circ} \mathrm{C}$, and the conversion factor was $58.0 \pm 1.8 \mathrm{mV} / \mathrm{pH}$ unit (Li and Nattie, 2002).

Histological analysis of cannula locations. After completing all experimental conditions, Fast Green was added to the aCSF and perfused into the RTN for $\sim 1 \mathrm{~h}$ (a time similar to the duration of a typical experiment) to evaluate the location of each cannula and the distribution of perfusate within the medulla. After the dye was perfused, the rat was deeply anesthetized and perfused transcardially through the left ventricle with a tissue fixative (Streck S.T.F. pH 7.4; Streck, Omaha, NE). After fixation, the brain was removed, flash frozen (Histofreeze; Thermo Fisher Scientific, Waltham, MA), and sectioned (40 $\mu \mathrm{m}$ thickness) with a cryostat maintained at $-20^{\circ} \mathrm{C}$ (Histostat; Reichart-Jung, NuBlock, Germany). Tissue sections were mounted on ultrastick glass slides (Gold Seal; BD Biosciences, San Jose, CA), stained with neutral red or cresyl violet, and 
serial sections were photographed using a digital camera (Orca-ER; Hamamatsu, Bridgewater, $\mathrm{NJ})$. The images were compared with photographic plates in a stereotaxic atlas to identify the location of the cannula in each section (Paxinos and Watson, 1998). Each cannula made a small hole in the tissue at the end of the cannula track, and the placement of the cannula was determined by identifying the section with the largest lucent cross-sectional area. The tissue stained by Fast Green was located directly adjacent to the tip of the guide cannula $(\sim 600 \mu \mathrm{m}$ in diameter), as in our previous studies in the RTN (Holleran et al., 2001; Hewitt et al., 2004; Parisian et al., 2004).

In vitro experiments: slice preparation for $\mathrm{pHi}$ measurements. Brain tissue slices were prepared from adult transgenic mice of either sex that express enhanced green fluorescent protein $(\mathrm{EGFP})$ in astrocytes $(\mathrm{FVB} / \mathrm{N} \times \mathrm{Tg}$ GFAPGFP; The Jackson Laboratory, Bar Harbor, ME) (Fig. $1 A)$. The mice were $3.6 \pm 0.6$ months of age at the time of study. Each animal was killed by rapid decapitation, and the brain was removed from the skull and submerged in chilled $\left(4^{\circ} \mathrm{C}\right)$ choline-substituted slicing solutions (described below) for 2-3 min. The caudal cerebellum was removed, and a tissue block extending from the obex caudally to the rostral portion of the pons was prepared. Transverse medullary slices (400 $\mu \mathrm{m}$ thick) were sectioned in chilled, oxygenated choline-substituted slicing solution using a vibratome (Series 1000; Pelco, Redding, CA). After sectioning, slices were transferred to a holding chamber containing oxygenated aCSF (described below) at room temperature.

Solutions for brain slice studies. The control aCSF contained the following (in $\mathrm{mm}$ ): 124 $\mathrm{NaCl}, 3 \mathrm{KCl}, 2.4 \mathrm{CaCl}_{2}, 1.3 \mathrm{MgSO}_{4}, 1.24$ $\mathrm{KH}_{2} \mathrm{PO}_{4}, 26 \mathrm{NaHCO}_{3}$, and 10 glucose. In solutions containing high concentrations of $\mathrm{K}^{+}$, $\mathrm{Na}^{+}$was reduced equally to keep the solution isosomotic. Except for the dose-response ex-

periments, 4-CIN was used at a final concentration of $100 \mu \mathrm{M}$ in the in vitro experiments. Before delivery to the tissue, all aCSF solutions were loaded into $60 \mathrm{ml}$ syringes, equilibrated with $95 \% \mathrm{O}_{2} / 5 \% \mathrm{CO}_{2}$ to maintain a $\mathrm{pH}$ of 7.48 at $37^{\circ} \mathrm{C}$, and heated to $37^{\circ} \mathrm{C}$ using a servo-controlled syringe heater block, in-line perfusion heater, and servo-controlled stage heater (models SW-707, SH-27B, TC-344B; Warner Instruments, New Haven, CT).

We used a modified aCSF solution to reduce cell death by reducing intracellular sodium and calcium and glutamatergic excitation. Therefore, the slicing solution contained no sodium (choline was substituted for sodium), reduced calcium, elevated magnesium, kynurenic acid (a glutamate receptor antagonist), and ascorbic acid as an antioxidant. Therefore, the choline-substituted slicing solution contained the following (in mM): 135 choline chloride, $1 \mathrm{KCl}, 0.5 \mathrm{CaCl}_{2}, 20 \mathrm{MgCl}_{2}, 1.4$ $\mathrm{NaH}_{2} \mathrm{PO}_{4}, 24$ choline bicarbonate, 20 kynurenic acid, 5 ascorbic acid, and 10 glucose. The choline substituted slicing solution was equilibrated with $95 \% \mathrm{O}_{2} / 5 \% \mathrm{CO}_{2}$ and chilled to $4^{\circ} \mathrm{C}$. The $\mathrm{pH}$ of the choline substituted slicing solution was $\sim 6.9$.

Measurement of pHi in brain slices. Because a proton is cotransported with the lactate anion across the cell membrane, pHi may be used as a surrogate measure of lactate transport (Schneider et al., 1993; Bröer et al., 1999). We used a pH-sensitive, red-shifted fluorescent probe, 5-(and-6)carboxyseminapthorfluorescein-1 (SNAFL) (Invitrogen, Carlsbad, $\mathrm{CA})$, for studies pHi. The emission spectra of SNAFL do not overlap the emission spectrum of EGFP in the mice we studied. Slices were loaded with $20 \mu \mathrm{M}$ SNAFL in control saline bubbled with $95 \% \mathrm{O}_{2} / 5 \% \mathrm{CO}_{2}$ for 45
B.

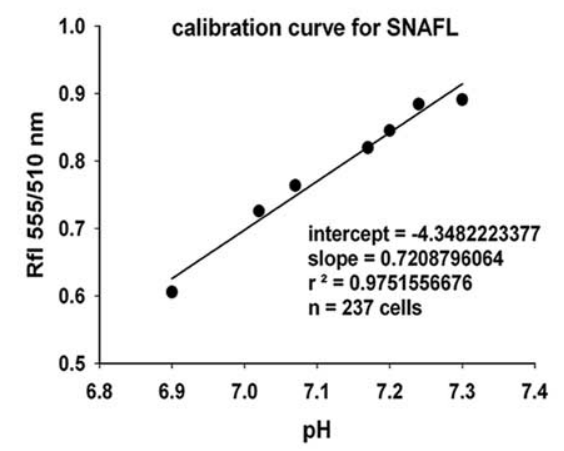

D.

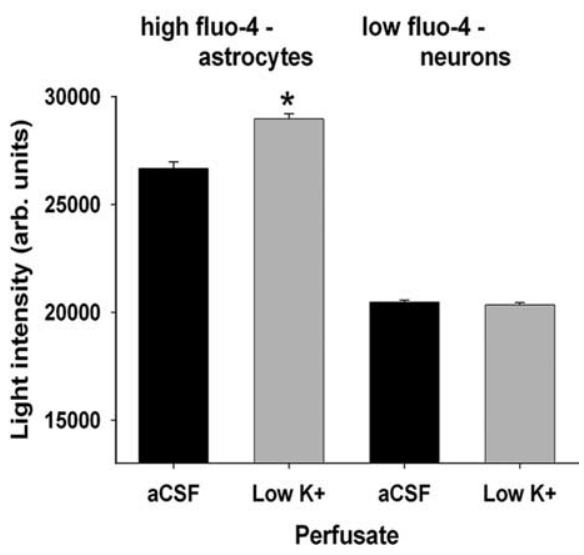

Perfusate

Figure 1. Expression of EGFP in astrocytes in brain slices prepared from transgenic mice is shown in $\boldsymbol{A}$. Scale bar, $30 \mu \mathrm{m}$. In $\boldsymbol{B}$, 列 to 7.3 , and the ratio of fluorescence at two excitation wave lengths $(550 \mathrm{and} 510 \mathrm{~nm}$ ) was determined. The calibration data were fit using a linear regression equation, because we used only a small pH range close to the pKa of SNAFL. Fluo-4 loading is shown in hepulation of Fluo-4 loaded cells was divided into two groups based on the intensity of Fluo-4 fluorescence in aCSF (D), only those cells with more intense uptake demonstrated increased intracellular $\mathrm{Ca}^{2+}$ levels after exposure to low-potassium solutions (Dallwig and Deitmer, 2002). ${ }^{*} p<0.05$.

min at room temperature. After loading with SNAFL, the slices were returned to a holding chamber, which contained aCSF equilibrated with $95 \% \mathrm{O}_{2} / 5 \% \mathrm{CO}_{2}$ at room temperature, for $30 \mathrm{~min}$ before experimentation. Intracellular $\mathrm{pH}$ was measured in single, SNAFL-loaded slices placed in a chamber that was continuously superfused with aCSF and mounted on the stage of a Nikon (Melville, NY) Eclipse TE200S inverted microscope. The $\mathrm{pHi}$ of individual cells was measured within discrete areas $\left(\sim 0.042 \mathrm{~mm}^{2}\right)$ of the medulla by alternately exciting the area with light from a $75 \mathrm{~W}$ xenon lamp (Chiu Technical Corporation, Kings Park, $\mathrm{NY})$ at $510 \pm 10$ and $555 \pm 10 \mathrm{~nm}$ wavelengths for $0.3-0.8 \mathrm{~s}$. The excitation filters were switched by a computer-controlled filter wheel (Lambda 10-2; Sutter Instrument, Novato, CA). The emitted fluorescence $(620 \mathrm{~nm})$ from the intracellular SNAFL was filtered using a $585 \mathrm{~nm}$ dichroic mirror (Chroma Technology, Bennington, VT), intensified, and measured with a cooled CCD camera (Orca ER; Hamamatsu Photonics, Hamamatsu City, Japan). The digital images were acquired and processed with Compix software (Compix Imaging Systems, Cranberry Township, PA).

The ratiometric values of $\mathrm{pHi}$ based on emitted SNAFL fluorescence were calibrated using the nigericin method (Boyarsky et al., 1988). The nigericin calibration solution contained the following (in $\mathrm{mM}$ ): $104 \mathrm{KCl}$, $2.4 \mathrm{CaCl}_{2}, 1.3 \mathrm{MgSO}_{4}, 1.24 \mathrm{KH}_{2} \mathrm{PO}_{4}, 10$ glucose, $25 \mathrm{~N}$-methyl-Dglucamine-HEPES, $25 \mathrm{~K}$-HEPES, and $1.6 \times 10^{-2}$ nigericin. A calibration curve was constructed by measuring the $555 / 510 \mathrm{~nm}$ ratio fluorescence (RFL) of cells $(n=237)$ exposed to nigericin containing calibration solutions of known $\mathrm{pH}$ ranging from 6.9 from $7.3 \mathrm{pH}$ units at $37^{\circ} \mathrm{C}$. These 
ratio measurements were fit by linear regression, and changes in $\mathrm{pHi}$ were calculated from the following calibration equation: $\mathrm{pHi}=\mathrm{RFL}+$ $4.34 / 0.721\left(r^{2}=0.98\right)$ (Fig. $\left.1 B\right)$. After each calibration, the entire perfusion apparatus was soaked and washed three times in $70 \%$ ethanol in water and subsequently rinsed repeatedly in distilled water to remove any retained nigericin.

Measurement of glucose uptake. We used EGFP(-) mice to study glucose uptake in brain slices. Brain slices containing the RTN were prepared from the medulla of adult mice as described above. Astrocytes were identified using a protocol in which brain slices were incubated in Fluo-4 $\mathrm{AM}\left(4 \mu \mathrm{M}\right.$; Invitrogen) in aCSF, and the extracellular $\mathrm{K}^{+}$was elevated to $5 \mathrm{~mm}$ for $45 \mathrm{~min}$ at room temperature (Dallwig and Deitmer, 2002). Astrocytes loaded using this method demonstrate $\mathrm{Ca}^{2+}$ oscillations when exposed to $\mathrm{K}^{+}$solutions containing nominal potassium (Fig. 1C) and colabel with glial fibrillary acidic protein (Dallwig and Deitmer, 2002). Neurons take up Fluo-4 as well. However, the staining was less intense compared with glia, and there were no intracellular $\mathrm{Ca}^{2+}$ oscillations when neurons were exposed to low-potassium solutions (Fig. $1 D)$. Fluo-4-loaded cells were visualized by exciting the fluorophore at $480 \pm 10 \mathrm{~nm}$ and filtering the emitted light $(535 \pm 10 \mathrm{~nm})$ through a 505 $\mathrm{nm}$ long-pass dichroic filter (Chroma Technology). Cells were defined as glia if they were Fluo- 4 positive and if the emitted light intensity after loading was above the mean light intensity level for the entire group of cells measured. The Fluo-4-negative group (putative neurons) was comprised of cells in which the intensity of emitted fluorescent light was below the mean intensity for the entire group of cells measured. That this Fluo-4 method accurately identified astrocytes and neurons in our studies was confirmed subsequently when we obtained mice expressing EGFP exclusively in astrocytes. The correspondence between high-intensity Fluo-4 fluorescence and the presence of EGFP in brain cells in these animals was virtually $1: 1$ (J. S. Erlichman, unpublished observations).

We used 2-[N-(7-nitrobenz-2-oxa-1,3-diazol-4-yl)amino]-2-deoxyglucose (2-NBDG), a fluorescent glucose tracer, to measure glucose uptake (Yamada et al., 2007). Regions of interest were defined by the somatic distribution of Fluo-4, and 2-NBDG uptake was subsequently measured within these regions. To measure 2-NBDG uptake, 2-NBDG $(15 \mu \mathrm{M})$ was added to aCSF, and this solution was used to perfuse the slice for $1 \mathrm{~min}$, after which time the solution was switched back to control aCSF and washed for $5 \mathrm{~min}$. The $5 \mathrm{~min}$ wash was necessary to remove free 2 -NBDG from the extracellular space. This procedure was repeated four times during each experiment and resulted in progressively greater loading with each 2-NBDG pulse. Between each loading/wash period, the fluorescent intensity of 2-NBDG was measured in each cell each minute for $3 \mathrm{~min}$ after the wash was completed. We determined in preliminary experiments that a $1 \mathrm{~min}$ loading period with the tracer was sufficient to generate an incremental increase in light intensity that yielded a satisfactory signal above baseline Fluo-4 fluorescence and would not saturate our camera after four periods of loading. Once the tissue was washed, the light intensity collected from subsequent images declined slowly, which likely reflected the degradation of phosphorylated 2-NBDG to a nonfluorescent metabolite (Yamada et al., 2000, 2007). The effect of inhibition of MCT2 with $100 \mu \mathrm{M}$ 4-CIN was assessed by comparing 2-NBDG uptake before 4-CIN treatment to 2-NBDG uptake after 4-CIN treatment in astrocytes and neurons. The 4-CIN was added to the perfusate between the second and third 2-NBDG treatment periods. In control studies, 2-NBDG uptake in astrocytes and neurons was measured after all four 2-NBDG treatment periods, and no 4-CIN treatment was given.

Data analysis and statistics. In the studies of intact animals, the type of perfusate delivered (aCSF or aCSF plus 4-CIN) and the $\mathrm{FICO}_{2}(0-8 \%)$ were used in a two-way repeated measures ANOVA (Systat 9.0; SPSS, Chicago, IL) to analyze the whole animal ventilatory responses. In the studies of pHe in intact animals, and the studies of $\mathrm{pHi}$ in brain slices, paired $t$ tests were used to compare steady-state $\mathrm{pH}$ values before and after treatment with 4-CIN. The analysis of the intensity of 2-NBDG uptake was conducted using a three-way ANOVA in which time of exposure (treatment 1,2,3, or 4) was a repeated, within subjects factor, and drug treatment (4-CIN vs no 4-CIN exposure) and cell type (astrocytes versus neurons) were between subjects factor. These data were also analyzed as a percentage of control 2-NBDG uptake intensity using a two-
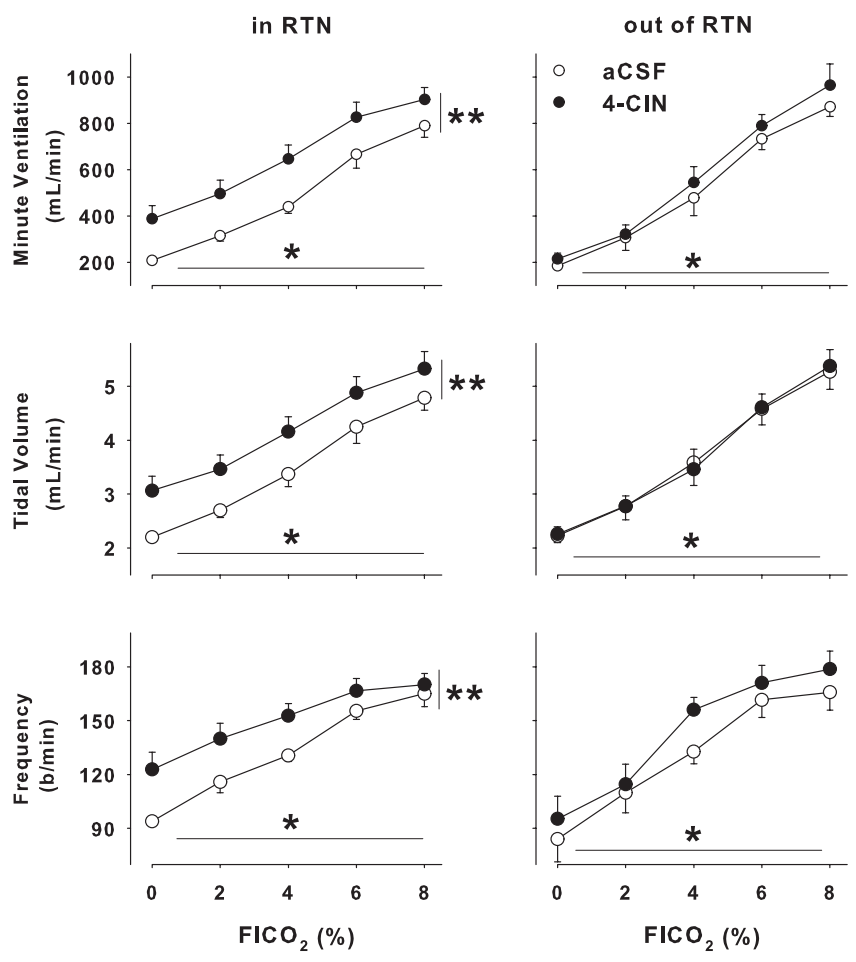

Figure 2. Ventilatory effects of 4-CIN perfusion unilaterally in the RTN. Minute ventilation, tidal volume, and frequency increased significantly as the fractional inspired $\mathrm{CO}_{2}$ increased ( $p<0.01$ for all three variables). In addition, focal unilateral perfusion of 4-CIN into the RTN increased minute ventilation, tidal volume, and frequency at all levels of $\mathrm{FICO}_{2}$ (left panels) $(p<0.01$ for all three variables; $n=9$ ). In contrast, 4-CIN perfused focally in the ventral medulla outside the area of the RTN did not change minute ventilation, tidal volume, or frequency $(n=3)$, although increasing the $\mathrm{FICO}_{2}$ increased these variables just as it had in the RTN treatment group.

way ANOVA in which time of exposure and drug treatment with or without 4-CIN were the two factors. When any of the ANOVAs indicated that significant differences existed among treatments, specific preplanned comparisons were made using $p$ values adjusted by the Bonferroni method. Values reported are the mean \pm SEM. $p$ values $\leq 0.05$ were considered statistically significant.

\section{Results}

Ventilatory effects of unilateral perfusion of RTN with 4-CIN

We studied hypercapnic ventilatory responses during focal unilateral perfusion of the RTN with aCSF or aCSF plus $100 \mu \mathrm{M}$ 4 -CIN in nine animals. The slope of the response of minute ventilation, tidal volume, and respiratory frequency was not changed by perfusion of the RTN with aCSF containing 4-CIN (no $\mathrm{CO}_{2}$ by drug interaction in the ANOVA) (Fig. 2, left). However, minute ventilation, tidal volume, and respiratory frequency were increased significantly for all three variables at every value of inspired $\mathrm{CO}_{2}$ during focal perfusion of aCSF plus 4-CIN compared with aCSF alone ( $p<0.01$ for all three variables). Moreover, ventilation, tidal volume, and respiratory frequency all increased significantly as the inspired $\mathrm{FICO}_{2}$ increased from 0 to $8 \%(p<$ 0.001 for all variables). Therefore, perfusion with 4 -CIN did not change the apparent $\mathrm{CO}_{2}$ sensitivity but did shift the ventilatory response curves up and to the left significantly for all three variables. The locations of all the cannulas in these nine animals fell within or closely adjacent to the RTN (Fig. 3, squares), which extends from the rostral end of the facial nucleus caudally to the rostral end of the nucleus ambiguus and mediolaterally between 
the pyramidal tract and the spinotrigeminal tract and is deep to the ventral surface 100-300 $\mu \mathrm{m}$ (Pearce et al., 1989).

To evaluate the specificity of the ventilatory effects of 4-CIN, three additional animals were cannulated in regions outside the RTN, and the ventilatory effects of 4-CIN were examined. In contrast to our findings in the RTN, unilateral perfusion of 4 -CIN in regions adjacent to the RTN had no significant effect on any of the measured ventilatory variables (Fig. 2, right panels). Hypercapnia still resulted in a significant increase in ventilation, but there was no effect of drug treatment on the ventilatory response to $\mathrm{CO}_{2}(p>0.05$, all three variables). The locations of the probes in these three animals are also shown in Figure 3 (triangles). Focal administration of $100 \mu \mathrm{M} 4$-CIN unilaterally within the RTN increased minute ventilation at all levels of inspired $\mathrm{CO}_{2}$, and focal administration of 4-CIN outside the RTN did not increase minute ventilation. Therefore, the effect of blocking neuronal lactate uptake on ventilation was specific to the RTN.

Effects of 4-CIN on pHe in the RTN Microinjection of aCSF plus 4-CIN into the RTN in awake rats resulted in a rapid and significant acid shift in $\mathrm{pHe}$ of $\sim 0.08 \pm 0.02 \mathrm{pH}$ units $(p<0.05)$. To compare the level of extracellular acidification associated with 4-CIN to the effect of inhaled $\mathrm{CO}_{2}$, we performed a separate set of experiments in which the $\mathrm{FICO}_{2}$ was increased to $5 \%$ while simultaneously measuring pHe in the RTN. The elevated inspired $\mathrm{CO}_{2}$ reduced the $\mathrm{pHe}$ in the RTN by $0.07 \pm 0.1 \mathrm{pH}$ units $(p<0.05)$, but the pHe change was similar to that observed with the 4-CIN injection alone. These results suggest that both treatments result in a similar stimulus to the acid-sensitive cells in the RTN, an area that is known to increase ventilation when acidified in similar studies in adult rats (Akilesh et al., 1997; Erlichman et al., 1998; Li et al., 1999; Li and Nattie, 2002). Administration of 4-CIN may have decreased pHe outside the RTN (we did not make these measurements), but these regions do not contain a density of chemosensory neurons sufficient to stimulate ventilation (Akilesh et al., 1997; Li et al., 1999), or the density of astrocytes may be too low to generate sufficient lactate (Erlichman et al., 2004).

\section{Effects of 4-CIN on pHi in the RTN}

We studied the effects of 4-CIN on pHi in $\operatorname{EGFP}(-)$ cells, which we defined as neurons, and in $\operatorname{EGFP}(+)$ cells, which we defined as astrocytes, in the region of the RTN in brain slices. Intracellular $\mathrm{pH}$ was measured using the ratiometric fluorescent dye SNAFL. Under control conditions, astrocytic pHi was $7.34 \pm 0.01$ in glia $(n=80)$. The addition of 4 -CIN to the perfusate resulted in a small, rapid, and dose-dependent decrease in $\mathrm{pHi}$ compared with control conditions (Fig. 4A) $(p<0.05 ; n=92)$. The peak acid shift in astrocytic pHi after $10 \mathrm{~min}$ exposure to $100 \mu \mathrm{M} 4$-CIN was small, $0.005 \pm 0.004 \mathrm{pH}$ units, but significant $(p<0.05)$ (Fig. $4 C)$. The reduction in $\mathrm{pHi}$ was significantly greater after exposure to $500 \mu \mathrm{M} 4$-CIN $(0.02 \pm 0.01 \mathrm{pH}$ units), although the absolute decline in pHi was still modest.

In neurons, the baseline control pHi was $7.27 \pm 0.01(n=32)$, and this value was significantly lower than the $\mathrm{pHi}$ in astrocytes $(p<0.01)$, just as we found previously (Nottingham et al., 2001). The neuronal pHi was, however, slightly more alkaline than our previous estimate of neuronal pHi (Ritucci et al., 2005). In contrast to astrocytes, which were very slightly acidified after exposure to 4-CIN, neurons were consistently alkalinized when exposed to $100 \mu \mathrm{M} 4$-CIN (peak pHi, $7.31 \pm 0.01 ; n=32 ; p<0.01$ ) (Fig. $4 B$ ). The intracellular alkalosis was reversed when 4 -CIN was removed from the perfusate. During the washout of 4-CIN, the neurons demonstrated a transient, acidifying undershoot (nadir of pHi, $7.26 \pm 0.009$ ) before returning to control values. The acidifying undershoot indicates that the cells had activated $\mathrm{pH}$ regulatory mechanisms (likely the $\mathrm{Cl}^{-} / \mathrm{HCO}_{3}^{-}$exchanger) during the alkalinizing phase of 4-CIN treatment in an attempt to regulate $\mathrm{pHi}$ toward the initial control value. The $\mathrm{pHi}$ values measured during exposure to 4 -CIN as well as the washout period were significantly different from control ( $p<0.001$ for both time 
A.

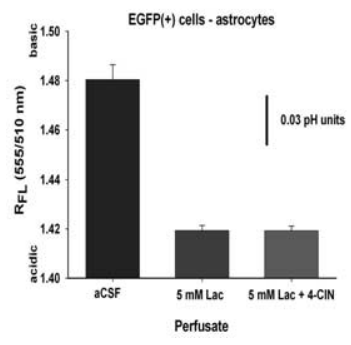

B.

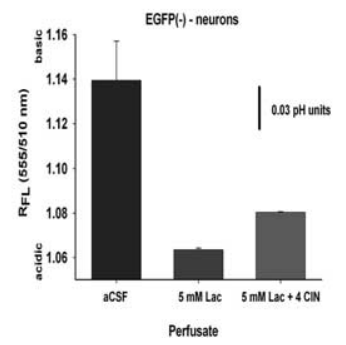

c.

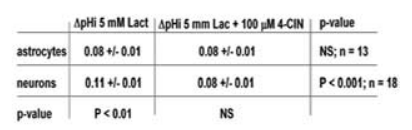

Figure 5. $\quad \boldsymbol{A}, \boldsymbol{B}$, Effects of $5 \mathrm{~mm}$ sodium lactate and $100 \mu \mathrm{m}$ 4-CIN on pHi in astrocytes $(\boldsymbol{A})$ and neurons $(\boldsymbol{B})$. The peak change in pHi from control before and after $100 \mu \mathrm{m}$ 4-CIN treatment is shown for each cell type in Calong with the $p$ values for comparisons between the control and test conditions and between cell types within each condition. NS, Not statistically significant.

equal concentrations of extracellular lactate were significantly different between the cell types $(p<0.01)$. The addition of $100 \mu \mathrm{M} 4$-CIN to the aCSF/lactate solution had little effect on the magnitude of the lactic acidosis in astrocytes (Fig. $5 C)(\Delta$ pHi 0.08 vs $0.08 ; n=13 ; p=0.83$ ), whereas the addition of $100 \mu \mathrm{M} 4$-CIN decreased the size of the shift in neuronal pHi by $\sim 20 \%$; the pHi difference between lactate alone and lactate plus $100 \mu \mathrm{M} 4$-CIN was statistically significant (Fig. $5 C)(\Delta$ pHi before 4-CIN, 0.11 vs $0.08 \mathrm{pH}$ units after 4 -CIN; $n=18 ; p<0.001)$. Therefore, 4 -CIN inhibited the movement of lactate into neurons but not into astrocytes, which is consistent with the hypothesis that lactate moves from astrocytes to neurons.
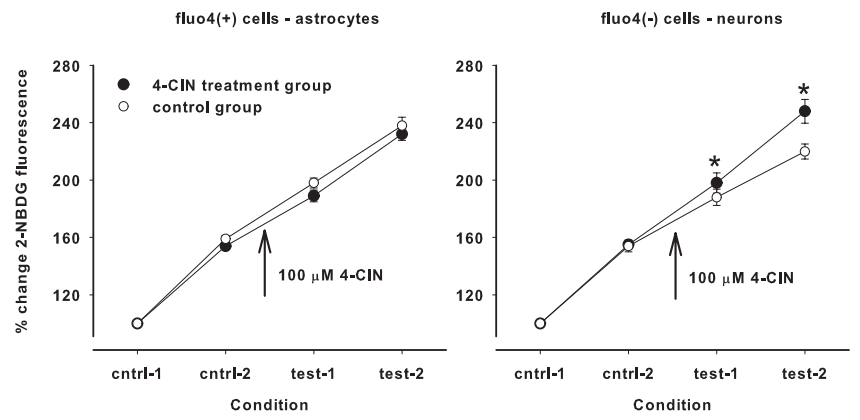

Figure 6. Uptake of 2-NBDG, expressed as a percentage of the first exposure period, increased in each subsequent loading period ( $p<0.001$ ). The comparison between control conditions (no 4-CIN treatment; open circles) and test conditions (100 $\mu$ m 4-CIN added between the second control period and the first test period; closed circles) are shown for astrocytes on the left and neurons on the right. Astrocytic loading of 2-NBDG was unaffected by 4-CIN treatment, but neuronal loading of 2-NBDG was significantly increased $\left({ }^{*} p<0.05\right)$ in each test condition.

points). Measurements of $\mathrm{pHi}$ alone cannot identify the form of the monocarboxylate being transported (i.e., lactate, pyruvate, or ketones), but it is likely that lactate was the predominant monocarboylate transported in our studies. The baseline extracellular lactate concentration in the CNS is $\sim 1-3 \mathrm{~mm}$ during resting conditions in vivo, and although the $K_{\mathrm{m}}$ of MCT2 for pyruvate is fivefold less than the $K_{\mathrm{m}}$ for lactate (Bröer et al., 1999), the pyruvate concentration in the cortex in vivo is $\sim 20$-fold less than the concentration of lactate (Homola et al., 2006). Lactate was likely the major monocarboxylate transported between cellular compartments, and therefore changes in lactate levels were the cause of the pHi changes that we measured. Mitochondrial monocarboxylate transporters in neurons can be inhibited by $4-\mathrm{CIN}$. Had this happened, we might have expected an increase in glycolysis and a decrease in neuronal $\mathrm{pHi}$. In fact, 4 -CIN treatment was associated with a slight alkaline shift in neuronal $\mathrm{pHi}$, and it seems unlikely that $100 \mu \mathrm{M} 4$-CIN inhibited mitochondrial MCTs.

\section{Effects of 5 mm lactate pulse on pHi in the RTN}

To assess the cellular specificity of lactate transport and the selectivity of the 4-CIN dose that we studied, we examined the effect of exogenous lactate $(5 \mathrm{~mm})$ on $\mathrm{pHi}$ in astrocytes and neurons in the presence or absence of $100 \mu \mathrm{M} 4$-CIN. The addition of sodium lactate to the aCSF solution created an inwardly direct lactate gradient and resulted in a decrease in pHi in all cells tested (Fig. $5 A, B)(n=31 ; p<0.001)$. The lactate pulse decreased $\mathrm{pHi}$ in astrocytes by $0.08 \pm 0.01 \mathrm{pH}$ units and decreased neuronal $\mathrm{pHi}$ by $0.11 \pm 0.01 \mathrm{pH}$ units. These changes in $\mathrm{pHi}$ after exposure to

\section{Loading of the glucose analog, 2-NBDG, in the RTN}

If lactate contributes significantly to the metabolism of neurons or astrocytes, then blocking lactate uptake using 4-CIN should result in a compensatory increase in glucose uptake. To assess this possibility, we measured the uptake of a fluorescent analog of glucose, 2-NBDG, before and after exposure to $100 \mu \mathrm{M} 4$-CIN. Both cell types demonstrated a significant increase in the accumulation of the fluorescent probe with repeated loading $(n=$ $426 ; p<0.001$ ). The uptake of $2-\mathrm{NBDG}$ was similar in neurons and astrocytes, but within astrocytes, the initial loading with 2-NBDG was a bit variable. Therefore, the intensity of 2-NBDG uptake in subsequent test periods was expressed as a percentage of the control values to facilitate comparisons of 2-NBDG uptake over time within each cell type (Fig. 6). The ANOVA of 2-NBDG uptake in astrocytes indicated that uptake increased significantly over time in all astrocytes tested $(p<0.001)$. However, the rate of uptake of 2-NBDG in astrocytes was unaffected by the addition of 4 -CIN to the perfusate between the second and third 2-NBDG exposure periods. The uptake of 2-NBDG by neurons also increased significantly over time, but the relative increase in 2-NBDG uptake was significantly greater in neurons that were treated with 4-CIN compared with neurons during the same 2-NBDG exposure periods that were not treated with 4-CIN ( $p<0.05$ for both the third and fourth 2-NBDG exposure periods). Therefore, 4-CIN treatment significantly increased 2-NBDG uptake in neurons but not in astrocytes.

\section{Discussion}

\section{Effects of MCT inhibition}

The ANLSH, as described originally (Pellerin and Magistretti, 1994; Magistretti and Pellerin, 1999), has been criticized: glutamate uptake by astrocytes is not coupled uniquely to anaerobic glycolysis, except possibly in cultured cells; it is not clear that there was net flux of lactate from astrocytes to neurons; and it has not been established that astrocytes are the source of lactate production or that neurons are the sink for lactate consumption; both cell types possess the enzymatic machinery for anaerobic and oxidative glycolysis (Chih et al., 2001; Hertz, 2004). Moreover, support for the hypothesis comes primarily from cultured cells rather than in vivo measurements (Kimmelberg, 2004). Subsequently, the ANLSH was modified to include just two ideas: glutamate uptake by astrocytes depends on anaerobic glycolysis, and neurons oxidize lactate produced by astrocytes (Pellerin and Magistretti, 2003).

Others have tested the first of these hypotheses in intact ani- 
mals (Lam et al., 2005), but we concerned ourselves with the second part of the ANLSH and tried to demonstrate the existence of a flux of lactate from astrocytes to neurons in vivo. Stimulation of central chemosensory neurons within the RTN increases minute ventilation ( $\mathrm{Li}$ and Nattie, 2002), and we reasoned that interruption of the lactate flux between astrocytes and neurons by selective inhibition of MCT2 using a low dose of 4-CIN should reduce $\mathrm{pHe}$ in the $\mathrm{RTN}$ as lactate accumulates in the extracellular space, which should stimulate ventilation. Therefore, we used the minute ventilation of intact animals to probe the effect of disrupting the lactate shuttle, and we measured pHe in the RTN before and after 4-CIN treatment to prove that 4-CIN stimulated ventilation by interrupting the flux of lactate. Focal administration of 4-CIN in the RTN did increase ventilation, and pHe decreased $\sim 0.8 \mathrm{pH}$ units, which indicates the presence of a lactate flux dependent on monocarboxylate transport in the RTN and provides support for the ANLSH. We cannot exclude the possibility of a nonspecific effect of 4-CIN on blood flow or some other aspect of metabolism or cell function in the RTN, which might result in extracellular acidosis, but we know of no such nonspecific effect of 4-CIN.

Nothing in the ventilatory and pHe measurements revealed which cellular elements were the source of lactate and which were the sink for lactate. Because lactate is transported 1:1 with a proton, an indirect means to investigate the direction of lactate transport is to follow the time course of changes in pHi using a fluorescent probe (Carpenter and Halestrap, 1994; Bröer et al., 1997, 1999). Exposure of brain slices to 4-CIN led to a dose-dependent acidosis in astrocytes and alkalosis in neurons. This is consistent with the hypothesis that 4-CIN blocked the uptake of lactate by neurons, and they were alkalinized slightly. In contrast, lactate accumulated in the extracellular space decreased the concentration gradient driving lactate out of astrocytes, lactate accumulated in astrocytes, and the pHi of astrocytes fell. We conclude, therefore, that lactate originates in astrocytes. Moreover, the greater acidification in neurons compared with astrocytes after the lactate pulse is consistent with a larger lactate concentration gradient in neurons, which favors movement of lactate into neurons where the lactate is consumed, a conclusion that supports the ANLSH (Pellerin and Magistretti, 2004).

\section{Glucose uptake in astrocytes and neurons in the RTN}

Astrocytes and neurons accumulated 2-NBDG at similar rates in the absence of 4-CIN (Wittendorp-Rechenmann et al., 2002; Nehlig et al., 2004). These investigators found that the amount of radiolabeled 2-deoxyglucose accumulated in neurons and astrocytes was approximately equivalent in brain slice studies. Similar uptake rates of 2-NBDG in astrocytes and neurons, nonetheless, represents a disproportionate astrocytic uptake of glucose, because the metabolic rate of astrocytes is less than the metabolic rate of neurons. Presumably much of the glucose taken up by astrocytes is shuttled to neurons in the form of lactate and contributes only modestly to energy consumption in the astrocytes (Rothman et al., 2003; Nehlig et al., 2004).

The uptake of 2-NBDG was increased in neurons in our study when monocarboxylate transport was impaired. This effect of 4-CIN on 2-NBDG uptake by neurons is similar, in general, to previous studies using radiolabeled 2-fluoro-2-deoxy-D-glucose to assess cell-specific metabolic rates in brain slices (Tanaka et al., 2004; Ogawa et al., 2005). These investigators found that 4-CIN treatment increased glucose uptake but only in some regions of the brain or only after periods of neuronal activation. We detected an increase in neuronal 2-NBDG uptake after 4-CIN treat- ment in unstimulated brain slices, indicating that neurons in the RTN metabolize some lactate even during resting conditions. Ventilation is a surrogate measure of pHe and a surrogate measure of changes in lactate levels in our study, and the slope of the ventilatory response to $\mathrm{CO}_{2}$ was not affected by 4-CIN treatment. This suggests that even during hypercapnic activation of the RTN when neuronal activity increases (Mulkey et al., 2004), the relative dependence of neurons on lactate was unchanged. Had the use of lactate increased in neurons as neuronal activity increased, we would have expected a steeper ventilatory response to $\mathrm{CO}_{2}$, because as the level of neuronal activity and astrocytic lactate release increased, the relative fall in $\mathrm{pHe}$ should have been greater and greater, but we did not see this. These results and those of others (Tanaka et al., 2004; Ogawa et al., 2005) raise two issues. First, dependence on lactate seems to vary from area to area within the brain. Presumably, this reflects the relative importance of glutamate as a neurotransmitter in each particular brain region but must also reflect the relative distribution of astrocytes within various areas of the brain. Second, lactate may be important in basal metabolism in some areas but may participate more importantly in activated states in others, which may also reflect the relative rates of basal and activated glutamate release. Our findings support the ANLSH in general, but metabolic dependence on the ANLSH may be heterogeneous throughout the brain, and we were not able to demonstrate any dependence of the magnitude of lactate shuttling on the magnitude of neuronal activation, at least in so far as we may infer that from measurements of ventilation.

\section{Implications for ventilatory central chemosensitivity}

There has been a long debate about the exact stimulus of central $\mathrm{CO}_{2}$ chemoreceptors (Winterstein, 1910; Jacobs, 1920; Putnam et al., 2004). Studies of arterial pH, pHi, pHe, and neuronal activity indicate that changes in $\mathrm{pHi}$ are a sufficient stimulus of central chemosensory neurons (Filosa et al., 2002; Wang et al., 2002; Putnam et al., 2004) but not a necessary stimulus. In addition, many of the putative ion channels (Putnam et al., 2004; Chernov et al., 2008) and neurotransmitter receptors (Loeschcke, 1982) thought to mediate chemosensitivity are sensitive to changes in $\mathrm{pHe}$, rather than pHi. Our measurements strongly suggest that $\mathrm{pHe}$ is also a sufficient stimulus of central chemosensors, because ventilation was increased when pHe decreased, although it seems likely that 4-CIN treatment alkalinized neuronal pHi. An alternative interpretation is that the increase in pHi in RTN neurons during MCT inhibition may have decreased activity of neurons alkalinized by 4-CIN treatment, but the extracellular acidosis extended beyond the direct effects of 4-CIN. Where this is the case, we might have expected ventilatory stimulation from this extended volume of extracellular acidosis after administration of 4-CIN just outside the RTN, but we did not see this in our control studies (Figs. 2, 3). Therefore, pHe may also be a sufficient stimulus, but like $\mathrm{pHi}$, not a necessary stimulus, because chemosensory neurons may be activated when pHe is held constant (Filosa et al., 2002; Wang et al., 2002; Ritucci et al., 2005). Central chemoreceptors probably have no necessary $\mathrm{pHi}$ or $\mathrm{pHe}$ stimulus but respond to the particular combination of $\mathrm{pHi}$ and $\mathrm{pHe}$ present in central chemosensory nuclei.

Students of the biology of astrocytes are fond of stating that astrocytes participate in information processing (Araque et al., 2001), although the evidence supporting such a statement in intact animals is almost nonexistent. The impact of inhibiting monocarboxylate transport on ventilation and $\mathrm{pH}$ in the RTN 
indicates that astrocytes actually determine, at least in part, the local $\mathrm{pH}$ and therefore the stimulus of central chemoreceptors in the RTN in intact animals. The distribution of astrocytes is heterogeneous in the CNS: astrocytes are densely distributed throughout the RTN, but the nucleus tractus solitarius (NTS), which also contains central chemosensory cells (Putnam et al., 2004), has relatively few astrocytes (Erlichman et al., 2004). It seems likely that the ANLSH is a significant factor setting $\mathrm{pHe}$ for chemosensory cells in the RTN, but not in the NTS. In this way, lactate transport and other astrocytic processes may make a significant, but anatomically variable contribution to the "information" processing that constitutes the central chemosensory stimuli (Erlichman et al., 2004). These ideas are consistent with focal measurements of $\mathrm{pHe}$ in the brainstem, which indicated that significant regional variation exists in pHe in the brainstem even when the $\mathrm{CO}_{2}$ stimulus is constant (Arita et al., 1989).

\section{References}

Akilesh MR, Kamper M, Li A, Nattie EE (1997) Effects of unilateral lesion of retrotrapezoid nucleus on breathing in awake rats. J Appl Physiol 82:469-479.

Araque A, Carmingoto G, Haydon PG (2001) Dynamic signaling between astrocytes and neurons. Annu Rev Physiol 63:795-813.

Arita H, Ichikawa K, Kuwana S, Kogo N (1989) Possible locations of $\mathrm{pH}$-dependent central chemoreceptors: intramedullary regions with acidic shift of extracellular fluid $\mathrm{pH}$ during hypercapnia. Brain Res 485:285-293.

Boyarsky G, Ganz MB, Sterzel RB, Boron WF (1988) pH regulation in single glomerular mesangial cells. I. Acid extrusion in the absence and presence of $\mathrm{HCO}_{3}{ }^{-}$. Am J Physiol 255:C844-C856.

Bröer S, Rahman B, Pellegri G, Pellerin L, Martin J-L, Verleysdonk S, Hamprecht B, Magistretti PJ (1997) Comparison of lactate transport in astroglial cells and monocarboxylate transporter 1 (MCT1) expressing Xenopus laevis oocytes. J Biol Chem 272:3009-30102.

Bröer S, Bröer A, Schneider H-P, Stegen C, Halestrap AP, Deitmer JW (1999) Characterization of the high-affinity monocarboxylate transporter MCT2 in Xenopus laevis oocytes. Biochem J 341:529-535.

Carpenter L, Halestrap AP (1994) The kinetics, substrate and inhibitor specificity of the lactate transporter of Ehrlich-Lettre tumor cells studied with the intracellular $\mathrm{pH}$ indicator BCECF. Biochem J 304:751-760.

Chernov MM, Leiter JC, Putnam RW (2008) A computer model of mammalian central $\mathrm{CO}_{2}$ chemoreception. Adv Exp Med Biol 605:301-305.

Chih C-P, Lipton P, Roberts Jr EL (2001) Do active cerebral neurons really use lactate rather than glucose? Trends Neurosci 24:573-578.

Dallwig R, Deitmer JW (2002) Cell-type specific calcium responses in acute rat hippocampal slices. J Neurosci Meth 116:77-87.

Debernardi R, Pierre K, Lengacher S, Magistretti PJ, Pellerin L (2003) Cellspecific expression pattern of monocarboxylate transporters in astrocytes and neurons observed in different mouse brain cortical cell cultures. J Neurosci Res 73:141-155.

Erlichman JS, Li A, Nattie EE (1998) Ventilatory effects of glial dysfunction in a rat brain stem chemoreceptor region. J Appl Physiol 85:1599-1604.

Erlichman JS, Cook A, Schwab MC, Budd TW, Leiter JC (2004) Heterogeneous patterns of $\mathrm{pH}$ regulation in glial cells in the dorsal and ventral medulla. Am J Physiol 286:R289-R302.

Filosa JA, Dean JB, Putnam RW (2002) Role of intracellular and extracellular $\mathrm{pH}$ in the chemosensitive response of rat locus coeruleus neurones. J Physiol (Lond) 541 2:493-509.

Gerhart EZ, Enerson BE, Zhdankina OY, Leino RL, Drewes LR (1997) Expression of monocarboxylate transporter MCT1 by brain endothelium and glia in adult and suckling rats. Am J Physiol 273:E207-E213.

Halestrap AP, Price NP (1999) The proton-linked monocarboxylate transporter (MCT) family: structure, function and regulation. Biochem J 343:281-299.

Hertz L (2004) The astrocyte-neuron lactate shuttle: a challenge of a challenge. J Cereb Blood Flow Metab 24:1241-1248.

Hewitt A, Barrie R, Graham M, Bogus K, Leiter JC, Erlichman JS (2004)
Ventilatory effects of gap junctions in the RTN in awake rats. Am J Physiol 287:R1407-R1418.

Holleran J, Babbie M, Erlichman JS (2001) The ventilatory effects of impaired glial function in a rat brainstem chemoreceptor region in the conscious rat. J Appl Physiol 90:1539-1547.

Homola A, Zoremba N, Šlais K, Kuhlen R, Syková E (2006) Changes in diffusion parameters, energy-related metabolites and glutamate in the rat cortex after transient hypoxia/ischemia. Neurosci Lett 404:137-142.

Jacobs MH (1920) The production of intracellular acidity by neutral and alkaline solutions containing carbon dioxide. Am J Physiol 53:457-463.

Kimmelberg HK (2004) The role of hypotheses in current research, illustrated by hypotheses on the possible role of astrocytes in energy metabolism and cerebral blood flow: from Newton to now. J Cereb Blood Flow Metab 24:1235-1239.

Lam TKT, Gutierrez-Juarez R, Pocai A, Rossetti L (2005) Regulation of blood glucose by hypothalamic pyruvate metabolism. Science 309:943-947.

Li A, Nattie EE (2002) $\mathrm{CO}_{2}$ dialysis in one chemoreceptor site, the RTN: stimulus intensity and sensitivity in the awake rat. Respir Physiol Neurobiol 133:11-22.

Li A, Randall M, Nattie EE (1999) $\mathrm{CO}_{2}$ microdialysis in retrotrapezoid nucleus of the rat increases breathing in wakefulness but not sleep. J Appl Physiol 87:910-919.

Loeschcke HH (1982) Central chemosensitivity and the reaction theory. J Physiol (Lond) 332:1-24.

Magistretti PJ, Pellerin L (1999) Astrocytes couple synaptic activity to glucose utilization in the brain. News Physiol Sci 14:177-182.

Manning Fox JE, Meredith D, Halestrap AP (2000) Characterisation of human monocarboxylate transporter 4 substantiates its role in lactic acid efflux from skeletal muscle. J Physiol (Lond) 529 2:285-293.

Mulkey DK, Stornetta RL, Weston MC, Simmons JR, Parker A, Bayliss DA, Guyenet PG (2004) Respiratory control by ventral surface chemoreceptor neurons in rats. Nat Neurosci 7:1360-1369.

Nehlig A, Wittendorp-Rechenmann E, Lam CD (2004) Selective uptake of $\left[{ }^{14} \mathrm{C}\right] 2$-deoxyglucose by neurons and astrocytes: high resolution microautoradiopraphic imaging by cellular ${ }^{14} \mathrm{C}$-trajectory combined with immunohistochemistry. J Cereb Blood Flow Metab 24:1004-1014.

Nottingham S, Leiter JC, Wages P, Buhay S, Erlichman JS (2001) Developmental changes in intracellular $\mathrm{pH}$ regulation in medullary neurons of the rat. Am J Physiol 281:R1940-R1951.

Ogawa M, Watabe H, Teramoto N, Miyake Y, Hayashi T, Iida H, Murata T, Magata Y (2005) Understanding of cerebral energy metabolism by dynamic living brain slice imaging system with $\left[{ }^{18} \mathrm{~F}\right] \mathrm{FDG}$. Neurosci Res 52:357-361.

Pappenheimer JR (1977) Sleep and respiration of rats during hypoxia. J Physiol (Lond) 266:191-207.

Pappenheimer JR, Fencl V, Heisey SR, Held D (1965) Role of cerebral fluids in control of respiration as studied in unanesthetized goats. Am J Physiol 208:436-450.

Parisian K, Wages P, Hewitt A, Leiter JC, Erlichman JS (2004) Ventilatory effects of gap junction blockade in the NTS in awake rats. Respir Physiol Neurobiol 142:127-143.

Paxinos G, Watson C (1998) The rat brain in stereotaxic coordinates, Ed 2. Sydney: Academic.

Pearce R, Stornetta RL, Guyenet P (1989) Retrotrapezoid nucleus in the rat. Neurosci Lett 101:138-142.

Pellerin L, Magistretti PJ (1994) Glutamate uptake into astrocytes stimulates aerobic glycolysis: a mechanism coupling neuronal activity to glucose utilization. Proc Natl Acad Sci USA 91:10625-10629.

Pellerin L, Magistretti PJ (2003) Food for thought: challenging the dogmas. J Cereb Blood Flow Metab 23:1282-1286.

Pellerin L, Magistretti PJ (2004) Empiricism and rationalism: two paths toward the same goal. J Cereb Blood Flow Metab 24:1240-1241.

Pierre K, Magistretti PJ, Pellerin L (2002) MCT2 is a major neuronal monocarboxylate transporter in the adult mouse brain. J Cereb Blood Flow Metab 22:586-595.

Poole RC, Halestrap AP (1997) Interaction of the erythrocyte lactate transporter (monocarboxylate transporter 1) with an integral $70-\mathrm{kDa}$ membrane glycoprotein of the immunoglobulin superfamily. J Biol Chem 272:14624-14628.

Putnam RW, Filosa JA, Ritucci NA (2004) Cellular mechanisms involved in $\mathrm{CO}_{2}$ and acid sensing in chemosensitive neurons. Am J Physiol 287:C1493-C1526. 
Ritucci NA, Erlichman JS, Leiter JC, Putnam RW (2005) Response of membrane potential $(\mathrm{Vm})$ and intracellular $\mathrm{pH}(\mathrm{pHi})$ to hypercapnia in neurons and astrocytes from rat retrotrapezoid nucleus (RTN). Am J Phsyiol 289:R851-R861.

Rothman DL, Behar KL, Hyder F, Shulman RG (2003) In vivo NMR studies of the glutamate neurotransmitter flux and neuroenergetics: implications for brain function. Annu Rev Physiol 65:401-427.

Schneider U, Poole RC, Halestrap AP, Grafe P (1993) Lactate-proton cotransport and its contribution to interstitial acidification during hypoxia in isolated rat spinal roots. Neuroscience 53:1153-1162.

Tanaka M, Nakamura F, Mizokawa S, Matsumura A, Matsumura K, Murata T, Shigematsu M, Kageyama K, Ochi H, Watanabe Y (2004) Role of lactate in the brain energy metabolism: revaled by bioradiography. Neurosci Res 48:13-20.

Wang W, Bradley SR, Richerson GB (2002) Quantification of the response of rat medullary raphe neurones to independent changes in $\mathrm{pH}_{\mathrm{o}}$ and $\mathrm{P}_{\mathrm{CO} 2}$. J Physiol (Lond) 540:951-970.

Winterstein H (1910) Das Überleben neugeborener Säugetiere bei künstlicher Durchspülung. Wien med Wochenschr 60:2274.

Wittendorp-Rechenmann E, Lam CD, Steibel F, Lasbennes F, Nehlig A (2002) High resolution tracer targeting combining microautoradiographic imaging by cellular ${ }^{14} \mathrm{C}$-trajectory with immunohistochemistry: a novel protocol to demonstrate metabolism of $\left[{ }^{14} \mathrm{C}\right] 2$-deoxyglucose by neurons and astrocytes. J Trace Microprobe Tech 20:505-515.

Yamada KA, Nakata M, Horimoto N, Saito M, Matsuoka H, Inagaki N (2000) Measurement of glucose uptake and intracellular calcium concentration in single, living, pancreatic $\beta$-cells. J Biol Chem 275:22278-22283.

Yamada KA, Saito M, Matsuoka H, Inagaki N (2007) A real-time method of imaging glucose uptake in single, living mammalian cells. Nat Protoc 2:753-762. 